\title{
Frequency of Zap-70 and CD38 in Newly Diagnosed Cases of B-Cell Chronic Lymphocytic Leukemia
}

\author{
Jahanzeb Khaliq ${ }^{1, *}$, Shafaq Samad ${ }^{2}$, Saima Siddique ${ }^{1,3}$, Mehjabeen $\operatorname{Imam}^{4}$, Tahir Sultan Shamsi ${ }^{1-4}$ \\ ${ }^{1}$ Department of Hematology, National Institute of Blood Disease \& Bone Marrow Transplantation, Karachi, Pakistan. \\ ${ }^{2}$ Department of Clinical Hematology, National Institute of Blood Disease \& Bone Marrow Transplantation, Karachi, Pakistan. \\ ${ }^{3}$ Department of Clinical Genetics, National Institute of Blood Disease \& Bone Marrow Transplantation, Karachi, Pakistan. \\ ${ }^{4}$ Department of Transplantation Immunology, National Institute of Blood Disease \& Bone Marrow Transplantation, Karachi, Pakistan.
}

\begin{abstract}
Introduction: Amongst all chronic lymphoproliferative disorders B-CLL is the most common. Clinical behavior of CLL is very variable and in order to identify the clinical spectrum there is a need for risk adaptive prognostic markers which will further facilitate in management strategy. Currently available molecular biomarkers ZAP-70 \& CD-38 have gain much interest in providing useful prognostic information in patients diagnosed as B-CLL.
\end{abstract}

Objective: To assess ZAP-70 and CD 38 positivity in patients diagnosed with B-CLL.

Material \& Methods: This was a cross sectional study conducted between Jan 2014 to July 2018 at NIBD, Karachi. Patients with B-CLL meeting the inclusion criteria were enrolled in the study. All patients gave informed consent. Immunophenotyping data analysis was done on FASC Calibur flowcytometry and Cell Quest software. We defined ZAP-70 and CD38 positivity as the presence of this marker on at least $20 \%$ and $30 \%$ of the B-CLL cells respectively. All required information was collected on pre designed Performa.

Results: Total of 101 patients diagnosed as B-Cell CLL were included in the study. Median age of enrolled patients was 64 years (range $43-85$ years). Majority of the patients i.e. 69 out of 101 (68.32\%) were males and the rest $32(31.68 \%)$ were female. ZAP-70 was positive in $2.97 \%$ of patients (03/101), while CD 38 positivity was detected in $33.66 \%(34 / 101)$.

Conclusion: ZAP-70 \& CD-38 positivity was present in $2.97 \%$ \& $33.66 \%$ of newly diagnosed B-CLL patients respectively. Screening for these biological markers in all newly diagnosed CLL patients will result in more dependable identification of disease clinical behavior.

Keywords: ZAP-70, CD-38, Chronic lymphocytic leukemia, Flowcytometry, Immunophenotyping, Prognosis.

\section{INTRODUCTION}

B Cell-Chronic Lymphocytic Leukemia (B-CLL), accounts for the most common chronic leukemia of adult in the western $[1,2]$. However, in Asian countries it is uncommon and accounts for less than 10\% cases [3]. Similarly, in Pakistan it has frequency of $9.7 \%$ amongst all the leukemia [4]. It is usually predominates in elderly with median age of onset of 65 years [5], however $10 \%$ of CLL patients are usually less than 55 years of age. The phenotypic presentation of this disease is extremely heterogeneous ranging from indolent disease to rapidly progressive requiring immediate treatment. Majority of patients have stage 0 or 1 at presentation (Rai/Binet staging system). New biological prognostic markers such as ZAP-70 \& CD-38 are independent predicable indicators of disease behavior [6]. ZAP-70 protein (defined as positive on at least $20 \%$ of B- cells), is actually a Zeta chain associated protein kinase. It has normal expression in $\mathrm{T}$ lymphocytes and natural killer cells, whereas on clonal B

*Address correspondence to this author at the Department of Hematology, National Institute of Blood Disease \& Bone Marrow Transplantation,

Karachi, Pakistan. E-mail: blacksuitdoc274@gmail.com lymphocytes it appears as an aberrant marker [7, 8]. Patients with ZAP-70 positivity at diagnosis have poor prognosis as it is associated with aggressive nature of disease requiring early management with chemotherapy, short progression free survival and decreased overall survival $[9,10]$.

CD-38 is a type II trans-membrane glycoprotein that functions as a receptor that induces proliferation $\&$ increases survival of CLL cells [11]. CD-38 positivity (defined as its presence on at least $30 \%$ of CLL cells) is an independent negative prognostic marker for clinical behavior in CLL $[12,13]$. These patients have more aggressive disease and also show diminished response to chemotherapy [11]. In a study conducted by Gogia A, et al. ZAP-70 \& CD-38 positivity was detected in 25 $\& 36 \%$ of Indian B cell CLL patients respectively [14]. On the other hand study conducted by Rozina et al. showed $13.5 \%$ ZAP-70 positive cases in Pakistani B-CLL patients [9].

Our study was designed to see the frequency of both CD-38 \& ZAP-70 positivity in our population, which would be helpful for risk stratification, patient counselling and compare it with local and international data. 


\section{MATERIAL \& METHODS}

Patients with B-Cell CLL meeting the inclusion criteria (All cases of B-Cell Chronic Lymphocytic Leukemia diagnosed on Flow cytometry at NIBD \& BMT, Both inpatient \& outpatient cases, Age $>40$ years $\&<90$ years, both gender) were enrolled in the study. An informed written consent was taken from all patients enrolled in study after explaining the purpose and procedure of the study. 3cc of peripheral blood was collected in EDTA tube. Whole blood was incubated at room temperature for 15 minutes with flourochrome conjugated antibodies including CD 5, CD 19, CD 20, CD 23, CD 79a, CD 11c, CD 25, FMC 7, CD 10, CD 3, CD 4, CD 8, CD 38, anti kappa light chain, anti lambda light chain \& ZAP 70. Using RBC lysing solution, RBCs were lysed. Leukocytes were washed with PBS solution twice. Tubes were then analyzed using BD FACS Caliber. Cell Quest Pro Software was used to acquire 10,000 events from each tube. Signals from each flour chrome were digitalized \& plotted on histograms of forward \& side scatter. Gate was applied at lymphocyte population \& analyzed for the presence of antibodies. All information was collected on predesigned Performa. Data was analyzed on statistically software SPSS version 17. Frequency \& percentage were computed for categorical data like CD-38 \& ZAP-70.

\section{RESULTS}

A total of 101 patients with B-Cell CLL were enrolled in the study. The median age of study population was 64 years (range 43-85 years). Majority of patients i.e., 69 out of 101 $(68.32 \%)$ were males (Fig. 1).

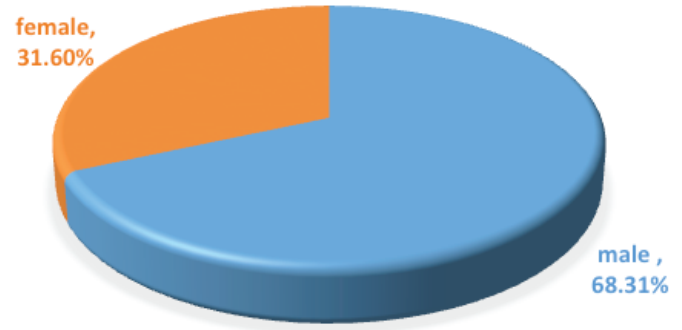

Fig. (1). Gender Distribution.

The frequency distribution of ZAP-70 and CD-38 positivity is presented in Table $\mathbf{1 .}$

Table 1. Frequency Distribution of Zap-70 and CD-38 Positivity.

\begin{tabular}{|c|c|c|}
\hline ZAP-70 POSITIVTY & $\begin{array}{c}\text { FREQUENCY } \\
\text { (n) }\end{array}$ & $\begin{array}{c}\text { PERCENTAGE } \\
\text { (\%) }\end{array}$ \\
\hline ABSENT & 98 & $97.03 \%$ \\
\hline PRESENT & 03 & $2.97 \%$ \\
\hline CD 38 & $\begin{array}{c}\text { FREQUENCY } \\
\text { (n) }\end{array}$ & $\begin{array}{c}\text { PERCENTAGE } \\
\mathbf{( \% )}\end{array}$ \\
\hline POSITIVTY & 67 & $66.34 \%$ \\
\hline ABSENT & 34 & $33.66 \%$ \\
\hline PRESENT & 101 & $100 \%$ \\
\hline TOTAL & \multicolumn{2}{|}{} \\
\hline
\end{tabular}

Only 03 (2.97\%) out of 101 patients were ZAP-70 positive. While CD-38 positivity was seen in 34 patients $(33.66 \%)$. Majority of the CD 38 positivity was seen in stage 3 and stage 4 disease i.e. $61.7 \%$ and $29.4 \%$ respectively (Table 2 ).

Table 2. Frequency of CD-38 According to the RAI Staging System of Disease.

\begin{tabular}{|c|c|c|}
\hline Stage & CD-38 Positive & CD-38 Negative \\
\hline Stage II & 03 & 34 \\
\hline Stage III & 21 & 18 \\
\hline Stage IV & 10 & 15 \\
\hline Total & 34 & 67 \\
\hline
\end{tabular}

Flow cytometric analysis showing positive expression of CD-38 and ZAP-70 on B-cell CLL markers CD5, CD19 (Fig. 2).
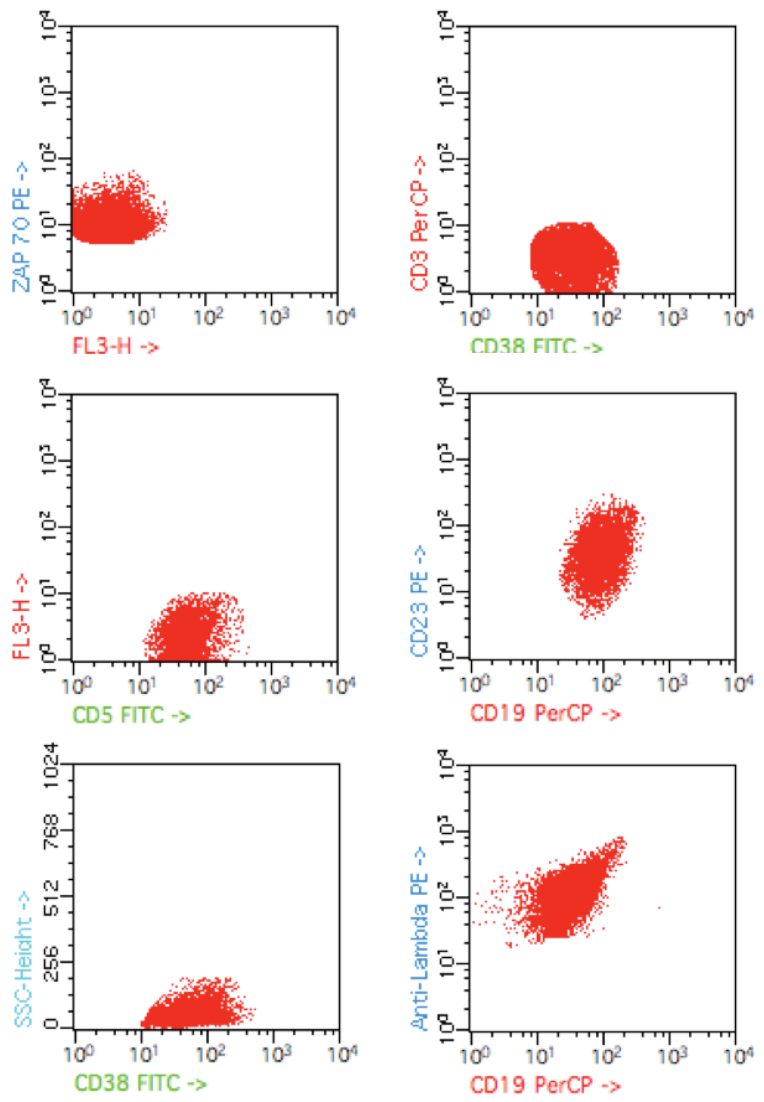

Fig. (2). Flow Cytometric Analysis Showing Positive Expression of CD-38 and ZAP-70 on B-Cell CLL Markers CD5, CD19.

\section{DISCUSSION}

A number of significantly new biological markers have been discern in B-Cell Chronic Lymphocytic Leukemia in the last two decades. Among them CD-38 and ZAP-70 expression in neoplastic B lymphocyte have independent predictable 
prognostic significance due their association with aggressive nature of disease, poor response to conventional chemotherapy, short progression free survival and decreased overall survival. ZAP-70 positivity in CLL has been reported from 36 to $57 \%$ in various studies. A Study conducted by Zeeshan et al. has showed Pakistani population to have $13.5 \%$ ZAP-70 positive CLL cases, whereas our study ZAP-70 positivity was found in only $2.97 \%$ of B-CLL cases. Study conducted by Gogia A, et al. from India revealed ZAP-70 positivity in $25 \%$ patients [14-17]. Another study from China by Qi RJ, et al. had reported the frequency of ZAP-70 to be $40.4 \%$ in all B-CLL cases. Screening for ZAP-70 at the time of diagnosis can be beneficial at the early identification of that subgroup of CLL patients who will be the candidate for early intensive management because of the aggressive nature of their disease.

In our study, CD-38 positivity was seen in 34 patients (33.66\%) as compared to various studies that have reported CD-38 positivity in CLL ranging from $29 \%$ to $60 \%$. Gogia A, et al. reported it to be $29 \%$ [14-17]. While Nihal et al. from Egypt reported it in $42.5 \%$ patients [18-20]. Majority of our patients with CD38 positivity had Rai stage 3 and 4 at the time of diagnosis.

Various studies have been published from our part of the world about the epidemiology, clinical and hematological markers and staging in CLL patients but even after the extensive search of literature we found that no study has been conducted so far in Pakistan that addresses the frequency of both CD-38 \& ZAP-70 simultaneously. Limitation of our study include small sample size. A larger group of cohort would be a better representative of ZAP-70 \& CD-38 prevalence in our population.

\section{CONCLUSION}

In our study, we concluded that ZAP-70 \& CD-38 positivity was present in $2.97 \%$ \& $33.66 \%$ of newly diagnosed B-CLL patients respectively. Moreover, CD-38 positivity was seen in more frequently in stage 3 and stage 4 of the disease at the time of diagnosis. So early screening for both CD38 and ZAP-70 in all newly diagnosed B-Cell CLL patients are surrogate markers of disease clinical spectrum which can guide about risk stratification, treatment outcome and overall survival.

\section{CONFLICT OF INTEREST}

Declared none.

\section{ACKNOWLEDGEMENTS}

Declared none.

\section{REFERENCES}

[1] Vroblová V, Smolej L, Vrbacky F, et al. Biological prognostic markers in chronic lymphocytic leukemia. Acta Medica (Hradec Kralove) 2009;52(1): 3-8.

DOI: $10.14712 / 18059694.2016 .99$

[2] Alikhan MB, Venkataraman G. Morphology and immunophenotype of chronic lymphocytic leukemia. In: Wiernik PH, Dutcher JP, Gertz MA, Eds. Neoplastic Diseases of the Blood. USA: Springer 2018; pp. 79-98.

DOI: $10.1007 / 978-3-319-64263-5 \_7$

[3] DeSantis CE, Lin CC, Mariotto AB, et al. Cancer treatment and survivorship statistics, 2014. CA Cancer J Clin 2014; 64(4): 252-71. DOI: 10.3322/caac. 21235

[4] Zeeshan R, Sultan S, Irfan SM, Kakar J, Hameed MA. Clinico-hematological profile of patients with B-chronic lymphoid leukemia in Pakistan. Asian Pac J Cancer Prev 2015; 16: 793-6. DOI: 10.7314/APJCP.2015.16.2.793

[5] Marti GE, Carter P, Abbasi F, et al. B-cell monoclonal lymphocytosis and B-cell abnormalities in the setting of familial B-cell chronic lymphocytic leukemia. Cytometry B Clin Cytom 2003; 52(1): 1-2. DOI: 10.1002/cyto.b.10013

[6] Sagatys EM, Zhang L. Clinical and laboratory prognostic indicators in chronic lymphocytic leukemia. Cancer Control 2012; 19(1): 18-25. DOI: 10.1177/107327481201900103

[7] Wang YH, Fan L, Xu W, Li JY. Detection methods of ZAP-70 in chronic lymphocytic leukemia. Clin Exp Med 2012; 12(2): 69-77. DOI: $10.1007 / \mathrm{s} 10238-011-0148-3$

[8] Chiorazzi N, Rai KR, Ferrarini M. Chronic lymphocytic leukemia. N Eng J Med 2005; 352(8): 804-15. DOI: $10.1056 /$ NEJMra041720

[9] Zeeshan R, Irfan SM, Sultan S, Bhimani S. ZAP-70 protein expression in B-cell chronic lymphoid leukemia: A single center experience from Pakistan. Asian Pac J Cancer Prev 2015; 16(4): 1587-90. DOI: 10.7314/APJCP.2015.16.4.1587

[10] Waheed R, Mahana N, Kassem N, El-Deeb SO. ZAP70 and CD38 as prognostic markers for chronic lymphocytic leukemia in Egyptian patients. Med J Cairo Univ 2015; 83(1): 627-32.

[11] Schroers R, Griesinger F, Trümper L, et al. Combined analysis of ZAP-70 and CD38 expression as a predictor of disease progression in B-cell chronic lymphocytic leukemia. Leukemia 2005; 19(5): 750. DOI: 10.1038/sj.leu.2403707

[12] Tinhofer I, Rubenzer G, Holler C, et al. Expression levels of CD38 in T cells predict course of disease in male patients with B-chronic lymphocytic leukemia. Blood 2006; 108(9): 2950-6. DOI: 10.1182/blood-2006-03-010553

[13] Del Poeta G, Maurillo L, Venditti A, et al. Clinical significance of CD38 expression in chronic lymphocytic leukemia. 
Blood 2001; 98(9): 2633-9. DOI: 10.1182/blood.V98.9.2633

[14] Gogia A, Sharma A, Raina V, Kumar L, Gupta R, Kumar R. Prevalence of ZAP-70 and CD 38 in Indian chronic lymphocytic leukemia patients. Indian J Cancer 2013; 50(4): 333. DOI: $10.4103 / 0019-509 X .123620$

[15] Rassenti LZ, Huynh L, Toy TL, et al. ZAP-70 compared with immunoglobulin heavy-chain gene mutation status as a predictor of disease progression in chronic lymphocytic leukemia. NEJM 2004; 351(9): 893-901. DOI: 10.1056/NEJMoa040857

[16] Hus I, Podhorecka M, Bojarska-Junak A, et al. The clinical significance of ZAP-70 and CD38 expression in B-cell chronic lymphocytic leukaemia. Ann Oncol 2006; 17(4): 683-90.

DOI: 10.1093/annonc/mdj120

[17] Junaid A, Rao PN, Adil MM. Chromosomal study for prognostic grouping in chronic lymphocytic leukemia. JCPSP 2011; 21(1): 19-22.

[18] El-Sharnouby JA, el-Shakankiri AA, Hendy OM, Ahmed LM, Taha AM. Significance of zeta-associated protein (ZAP-70) and CD38 expression in chronic lymphocytic leukemia. Egypt J Immunol 2006; 13(2): 69-84.

[19] Qi RJ, Zhang PH, Qiu LG, et al. Clinical significance of ZAP-70 protein expression in chronic lymphocytic leukemia/small lymphocytic lymphoma. Zhonghua Bing Li Xue Za Zhi 2009; 38(5): 329-32.

[20] El-Kinawy NS, Sharaf HM, El-Hamid M. Prognostic significance of del 17p, ZAP-70 and CD38 as independent indicators for B-CLL: Correlation to response to treatment and disease outcome. Egypt J Med Hum Genetics 2012; 13: 173-81. DOI: 10.1016/j.ejmhg.2012.03.007 